\title{
Not Always a Nail in the Coffin! Brainstem Lesions After Traumatic Brain Injury
}

\author{
Kristine H. O'Phelan ${ }^{*}$ (D)
}

(C) 2021 Springer Science+Business Media, LLC, part of Springer Nature and Neurocritical Care Society

If we have the humility to question our presumptions, we can save lives.

Traumatic brain injury (TBI) is a common occurrence, and it can be economically costly and life changing. Several demographics, physiologic data, and imaging characteristics have been identified as important determinants of outcomes after TBI. These include age, pupillary function, early Glasgow Coma Scale score, hypotension, and hypoxia. Other important factors have been identified and are incorporated into the International Mission for Prognosis and Analysis of Clinical Trials in TBI prognostic model, which includes computed tomography (CT) classification. However, the CT portion of the International Mission for Prognosis and Analysis of Clinical Trials in TBI model does not specifically include brainstem findings [1]. Historically, clinicians have considered any structural damage to the brainstem to be irreversible and a marker for poor clinical prognosis despite a lack of carefully focused research on the topic.

In their article "Prognostic Value of Hemorrhagic Brainstem Injury on Early Computed Tomography: A TRACK-TBI Study" the authors [2] present detailed analysis of early $\mathrm{CT}$ scans performed during routine clinical care in patients prospectively enrolled in the multicenter Transforming Research and Clinical Knowledge in TBI study. Their goal was to determine the contribution of hemorrhagic brainstem lesions seen on early noncontrast head CT to clinical outcome assessed at 6 months post injury. The authors compare the functional outcome of patients with hemorrhagic brainstem lesions with that of age-, sex-, and TBI severity-matched patients without

\footnotetext{
*Correspondence: kophelan@med.miami.edu

Miller School of Medicine, University of Miami, Miami, FL, USA

This article is related to the original article available at https://link.sprin ger.com/article/10.1007/s12028-021-01263-8.
}

brainstem lesions. They found no worsened outcome at 6 months post injury unless the lesions surpassed the threshold of $1 \mathrm{~cm}^{3}$. This work has several important strengths. First and foremost, it demonstrates that favorable outcomes are possible in patients with structural brainstem lesions seen on early imaging after TBI. Additionally, the incorporation of brainstem findings on $\mathrm{CT}$ into a prognostic model is valuable and may improve future tools for prognostication.

Some important limitations should be discussed. This study analyzed noncontrast CT scans performed during the first $48 \mathrm{~h}$ after admission. CT imaging was chosen presumably because it is routinely performed during clinical care. It is true that magnetic resonance imaging would provide a more accurate characterization of the exact nature and location of the lesions and improve on the ability to classify them as either traumatic axonal injury, contusions, or Duret hemorrhages. Perhaps magnetic resonance imaging could be incorporated in future protocols. Finally, 7 of 29 patients died after withdrawal of life-sustaining therapies (WOLST) before the 6-month outcome measure was reached. WOLST is the leading cause of death in patients who die after severe TBI [3]. Those who are assumed to have devastating brain injury are most likely to undergo early termination of supportive therapies [4]. The impact of WOLST on mortality in this study must be considered and may affect patients with brainstem hemorrhages more than those without brainstem hemorrhages. The early termination of treatment based on an inaccurate prediction of prognosis leads to rampant therapeutic nihilism in this population.

The integration of these findings into our clinical care will help us improve our prognostication and avoid early termination of aggressive support in patients who may recover and have a favorable outcome after TBI.

\section{Springer}


Source of Support

No funding was provided for this work.

\section{Conflicts of interest}

The author declares no conflicts of interest.

\section{Publisher's Note}

Springer Nature remains neutral with regard to jurisdictional claims in published maps and institutional affiliations.

Received: 20 April 2021 Accepted: 21 April 2021

Published online: 26 July 2021

\section{References}

1. Panczykowski DM, Puccio AM, et al. Prospective independent validation of impact modeling as a prognostic tool in severe traumatic brain injury. J Neurotrauma. 2011;29:47-52.

2. Williams JR, Nieblas-Bedolla E, et al. Prognostic value of hemorrhagic brainstem injury on early computed tomography: a TRACK-TBI study. Neurocrit Care. 2021. https://doi.org/10.1007/s12028-021-01263-8.

3. Leblanc G, Boutin A, et al. Incidence and impact of withdrawal of lifesustaining therapies in clinical trials of severe traumatic brain injury: a systematic review. Clin Trials. 2018;15(4):398-412.

4. Lazaridis C. Withdrawal of life-sustaining treatments in perceived devastating brain injury: the key role of uncertainty. Neurocrit Care. 2019;30(1):33-41. 